\title{
Identifying genetic risk variants for coronary heart disease in familial hypercholesterolemia: an extreme genetics approach
}

Jorie Versmissen ${ }^{1,31}$, Daniëlla M Oosterveer ${ }^{1,31}$, Mojgan Yazdanpanah ${ }^{1,31}$, Abbas Dehghan ${ }^{2}$, Hilma Hólm ${ }^{3}$, Jeanette Erdman ${ }^{4}$, Yurii S Aulchenko, ${ }^{2,5}$, Gudmar Thorleifsson ${ }^{3}$, Heribert Schunkert ${ }^{4}$, Roeland Huijgen ${ }^{6}$, Ranitha Vongpromek ${ }^{1}$, André G Uitterlinden ${ }^{1,2}$, Joep C Defesche ${ }^{6}$, Cornelia M van Duijn ${ }^{2}$, Monique Mulder ${ }^{1}$, Tony Dadd ${ }^{7}$, Hróbjartur D Karlsson ${ }^{8}$, Jose Ordovas ${ }^{9}$, Iris Kindt ${ }^{10}$, Amelia Jarman ${ }^{7}$, Albert Hofman ${ }^{2}$, Leonie van Vark-van der Zee ${ }^{1}$, Adriana C Blommesteijn-Touw ${ }^{1}$, Jaap Kwekkeboom ${ }^{11}$, Anho H Liem ${ }^{12}$, Frans J van der Ouderaa ${ }^{13}$, Sebastiano Calandra ${ }^{14}$, Stefano Bertolini ${ }^{15}$, Maurizio Averna ${ }^{16}$, Gisle Langslet ${ }^{17}$, Leiv Ose ${ }^{17}$, Emilio Ros ${ }^{18,19}$, Fátima Almagro ${ }^{20}$, Peter W de Leeuw ${ }^{21}$, Fernando Civeira ${ }^{22}$, Luis Masana ${ }^{23}$, Xavier Pintó ${ }^{24}$, Maarten L Simoons ${ }^{25}$, Arend FL Schinkel ${ }^{1,25}$, Martin R Green ${ }^{7}$, Aeilko H Zwinderman ${ }^{26}$, Keith J Johnson ${ }^{27}$, Arne Schaefer ${ }^{28}$, Andrew Neil ${ }^{29}$, Jacqueline CM Witteman ${ }^{2}$, Steve E Humphries ${ }^{30}$, John JP Kastelein ${ }^{6}$ and Eric JG Sijbrands ${ }^{\star, 1}$

Mutations in the low-density lipoprotein receptor (LDLR) gene cause familial hypercholesterolemia (FH), a disorder characterized by coronary heart disease (CHD) at young age. We aimed to apply an extreme sampling method to enhance the statistical power to identify novel genetic risk variants for CHD in individuals with FH. We selected cases and controls with an extreme contrast in CHD risk from $17000 \mathrm{FH}$ patients from the Netherlands, whose functional LDLR mutation was unequivocally established. The genome-wide association (GWA) study was performed on 249 very young FH cases with CHD and 217 old FH controls without CHD (above 65 years for males and 70 years of age for females) using the Illumina HumanHap550K chip. In the next stage, two independent samples (one from the Netherlands and one from Italy, Norway, Spain, and the United Kingdom) of FH patients were used as replication samples. In the initial GWA analysis, we identified 29 independent single nucleotide polymorphisms (SNPs) with suggestive associations with premature $\mathrm{CHD}\left(P<1 \times 10^{-4}\right)$. We examined the association of these SNPs with CHD risk in the replication samples. After Bonferroni correction, none of the SNPs either replicated or reached genome-wide significance after combining the discovery and replication samples. Therefore, we conclude that the genetics of CHD risk in FH is complex and even applying an 'extreme genetics' approach we did not identify new genetic risk variants. Most likely, this method is not as effective in leveraging effect size as anticipated, and may, therefore, not lead to significant gains in statistical power.

European Journal of Human Genetics (2015) 23, 381-387; doi:10.1038/ejhg.2014.101; published online 11 June 2014

\section{INTRODUCTION}

Coronary heart disease (CHD) is one of the leading causes of death. ${ }^{1}$ Multiple genetic risk variants with small to moderate effects on the susceptibility to $\mathrm{CHD}$ have been identified in genome-wide association (GWA) studies; however, these variants explain only a small fraction of the heritable component of the risk of CHD.,3

${ }^{1}$ Department of Internal Medicine, Erasmus University Medical Center, Rotterdam, The Netherlands; ${ }^{2}$ Department of Epidemiology, Erasmus University Medical Center, Rotterdam, The Netherlands; ${ }^{3}$ deCODE Genetics, Reykjavik, Iceland; ${ }^{4}$ Deutsches Hertzzentrum München, Technische Universität München, München, Germany; ${ }^{5}$ Novosibirsk State University, Novosibirsk, Russia; ${ }^{6}$ Department of Vascular Medicine, Amsterdam Medical Center, Amsterdam, The Netherlands; ${ }^{7}$ Unilever R\&D, Colworth Science Park, Sharnbrook, UK; ${ }^{8}$ Icelandic Heart Association and Department of Cardiology, Landspitali-University Hospital, Reykjavik, Iceland; ${ }^{9}$ Nutrition and Genomics Laboratory, Jean Mayer USDA HNRCA at Tufts University, Boston, MA, USA; ${ }^{10}$ Stichting Opsporing Erfelijke Hypercholesterolemie, Amsterdam, The Netherlands; ${ }^{11}$ Department of Gastroenterology and Hepatology, Erasmus University Medical Centre, Rotterdam, The Netherlands; ${ }^{12}$ Department of Cardiology, Sint Franciscus Gasthuis Hospital, Rotterdam, The Netherlands; ${ }^{13}$ Netherlands Consortium for Healthy Ageing, Leiden University Medical Center, Leiden, The Netherlands; ${ }^{14}$ Department of Biomedical Sciences, University of Modena and Reggio Emilia, Modena, Italy; ${ }^{15}$ Department of Internal Medicine, University of Genova, Genova, Italy; ${ }^{16}$ Department of Internal Medicine and Medical Specialties, University of Palermo, Palermo, Italy; ${ }^{17}$ Lipid Clinic, Oslo University Hospital, Oslo, Norway; ${ }^{18}$ Lipid Clinic, Endocrinology and Nutrition Service, Institut d’Investigacions Biomèdiques August Pi Sunyer (IDIBAPS), Hospital Clínic, Barcelona, Spain; ${ }^{19} \mathrm{CIBER}$ Fisiopatología de la Obesidad y Nutrición (CIBERobn), Instituto de Salud Carlos III (ISCIII), Madrid, Spain; ${ }^{20}$ Hospital de Guipúzcoa, San Sebastián, Guipúzcoa, España; ${ }^{21}$ Department of Internal Medicine, Maastricht University Medical Center, Maastricht, The Netherlands; ${ }^{22}$ Lipids Unit and Molecular Research Unit, Hospital Universitario Miguel Servet, Zaragoza, Spain; ${ }^{2}$ Unitat de Recerca en Lípids i Arteriosclerosi (URLA), Hospital Universitari Sant Joan de Reus, Reus, Spain; ${ }^{24}$ Unitat de Lípids i Arteriosclerosi, Servei de Medicina Interna, Hospital Universitari de Bellvitge, Barcelona, Spain; ${ }^{25}$ Department of Cardiology, Erasmus University Medical Center, Rotterdam, The Netherlands; ${ }^{26}$ Department of Clinical Epidemiology and Biostatistics, Amsterdam Medical Center, Amsterdam, The Netherlands; ${ }^{27}$ Novartis Insitutes of Biomedical Research, Cambridge, MA, USA; ${ }^{28}$ Institute of Clinical Molecular Biology, Christian-Albrechts-University, Kiel, Germany; ${ }^{29}$ Oxford Centre for Diabetes, Endocrinology and Metabolism, University of Oxford, Oxford, UK; ${ }^{30}$ Cardiovascular Genetics, BHF Laboratories, Institute Cardiovascular Science, University College London, London, UK

*Correspondence: Professor EJG Sijbrands, Vascular Medicine, Erasmus Medical Centre, Dr Molewaterplein 10, Rotterdam 3015 GD, The Netherlands. Tel: +31 10 0622569510 ; Fax: +31 1070336 39; E-mail: e.sijbrands@erasmusmc.nl

${ }^{31}$ These authors contributed equally to this work.

Received 5 May 2013; revised 12 April 2014; accepted 26 April 2014; published online 11 June 2014 
Therefore, many genetic variants remain to be discovered. Among the discovered genes, many are related to lipid metabolism. ${ }^{4,5}$ New study designs will be necessary for uncovering additional associated variants. Selecting the populations who already have high lipid levels are well suited to search for genes that increase the risk of CHD beyond dyslipidemia. ${ }^{6}$ Therefore, we performed a GWA study in a selected sample of familial hypercholesterolemia (FH) patients having severe hypercholesterolemia caused by mutations in the lowdensity lipoprotein receptor (LDLR) gene (MIM 143890). ${ }^{7}$

Traditional GWA studies are performed on extremely large sample sizes including tens of thousands of individuals. ${ }^{8}$ In contrast, we used an extreme genetics approach to enhance the statistical power. In this method, only the individuals with an extreme of the phenotype are genotyped. In our study, we genotyped FH patients who had CHD at very young age as cases, and elderly patients who, despite their high lowdensity lipoprotein (LDL) cholesterol level, had not experienced CHD as controls. We hypothesized that using this design would enhance the identification of additional genetic risk variants of $\mathrm{CHD}$ in $\mathrm{FH}$.

\section{MATERIALS AND METHODS}

The schematic study design is shown in Figure 1. We performed the study in two stages. Stage I included a GWA study in the Dutch 'Association of CHD Risk in a Genome-wide Old-versus-young Setting' (ARGOS) sample. Stage II consisted of genotyping of a second case-control sample, refer to as Mutations Associated with Risk of Cardiovascular disease in volunteers with Hypercholesterolemia (MARCH) and a large FH cohort, refer to as FH Follow-up (FHFU). All patients were of Caucasian descent. In ARGOS, this was confirmed by multi-dimensional scaling of identical-by-state pairwise distances. All patients gave informed consent and the local ethics committees approved the protocol.

\section{Description of the populations}

Gene-finding stage

ARGOS. The ARGOS sample consists of 500 patients, who were selected from 17000 Dutch FH patients with a mutation in the LDLR gene. They were recruited in the Netherlands by the nationwide molecular screening program of the 'Stichting Opsporing Erfelijke Familiare Hypercholesterolemie.' Phenotypic data (including CHD events) were acquired from general practitioners and by reviewing medical records at the lipid and cardiologic clinics. We selected the 264 youngest patients with premature CHD and the 236 oldest patients without any CHD, stratified for sex. The maximum age of the female cases was 60 years and that of the male cases 45 years. The minimum age of the controls was 65 years for males and 70 years for females. First and second degree family members were excluded. CHD was defined as

\begin{tabular}{|c|c|c|}
\hline \multicolumn{3}{|c|}{$\begin{array}{l}\text { ARGOS STAGE I } \\
249 \text { Youngest FH Patients with CHD } \\
217 \text { Elderly FH Patients without CHD }\end{array}$} \\
\hline Illumina $550 \mathrm{~K}$ & $29 S N P$ & $p<10^{-4}$ \\
\hline $\begin{array}{l}\text { MARCH } \\
190 \text { Cases } \\
223 \text { Controls }\end{array}$ & $\begin{array}{l}\text { FHFU } \\
554 \text { Cases } \\
1468 \text { Controls }\end{array}$ & STAGE II \\
\hline $\begin{array}{l}\text { META-ANAI } \\
* \text { MARCH+ FI } \\
* \text { ARGOS + M }\end{array}$ & $\begin{array}{l}\text { SIS } \\
\text { FU } \\
\text { RCH+ FHFU }\end{array}$ & \\
\hline
\end{tabular}

Figure 1 Study design. Abbreviations: CHD, coronary heart disease; FH, familial hypercholesterolemia; GWA, genome-wide association; SNP, single nucleotide polymorphism. the presence of at least one of the following: (i) myocardial infarction (MI), proved by at least two of the following: (a) classical symptoms ( $>15 \mathrm{~min}$ ), (b) specific abnormalities on electrocardiography, and (c) elevated cardiac enzymes ( $>2 \times$ upper limit of normal); (ii) percutaneous coronary intervention or other invasive procedures; (iii) coronary artery bypass grafting (CABG). Patients with angina pectoris were excluded because in the majority of cases this diagnosis could not be assured by objective data.

MARCH. The MARCH study group consisted of $413 \mathrm{FH}$ patients ( 190 cases, 223 controls), from Italy, Norway, Spain, and the United Kingdom. A few patients were born in another country but all were Caucasian. All patients had clinically proven $\mathrm{FH}$ and a mutation in either the $L D L R$ or the $A P O B$ gene. The maximum age of the female cases was 59 and that of the male cases 45 years. The minimum age of the controls was 50 years for males and 60 for females. For the cases in this cohort, the same CHD definition was applied as described above for the ARGOS sample with the addition of (iv) angina pectoris (AP), as this phenotype was accurately addressed in this cohort AP was diagnosed as classical symptoms in combination with at least one unequivocal positive result of one of the following: (a) exercise test, (b) nuclear scintigram, (c) dobutamine stress ultrasound, or (d) $>70 \%$ stenosis on a coronary angiogram. The controls had no manifest CHD.

FHFU study. The second replication cohort consisted of Dutch clinically proven heterozygous $\mathrm{FH}$ patients who were recruited from 27 lipid clinics in the Netherlands between 1989 and 2002. ${ }^{10,11}$ For the cases in this cohort, the same CHD definition was applied as described above for the MARCH sample. The controls had no manifest CHD. The DNA of 2073 unrelated patients was available for the present analysis. A total of $51 \mathrm{FH}$ patients had already been included in the ARGOS sample and were, therefore, removed from the FHFU group, leaving 2022 DNA samples for analyses.

Additional cohorts. In addition to these three FH cohorts, selected single nucleotide polymorphisms (SNPs) were also genotyped in population-based cohorts that have been described in detail previously: The Rotterdam Study ( $n=5207$, a cohort of elderly inhabitants of a suburb of Rotterdam, from which we selected patients with prevalent CHD to ascertain that CHD occurred at relatively young age; 777 CHD cases), deCODE $(n=12848$, selected from the national Islandic deCODE database; 726 cases with premature MI) and GerMIFSII $(n=2520$, a German cohort with 1222 proven MI cases before the age of 60$).{ }^{12-14}$

\section{Genotyping in ARGOS}

The samples of participants of the ARGOS group were assayed with Illumina Infinium HumanHap550K Chips (Illumina, San Diego, CA, USA) at Erasmus University Medical Center in Rotterdam, the Netherlands. Samples were processed according to the Illumina Infinium II manual. In brief, each sample was whole-genome amplified, fragmented, precipitated, and resuspended in the appropriate hybridization buffer. After hybridization, these denatured samples were processed for the single-base extension reaction and were stained and imaged on an Illumina Bead Array Reader. Normalized bead-intensity data obtained for each sample were loaded into the Illumina Beadstudio software where the fluorescent intensities were converted into SNP genotypes. Using Quanto software (http://biostats.usc.edu/software) considering ARGOS as a conventional case-control study and assuming large effect sizes owing to only genotyping the phenotypic extremes, we estimated that had $>80 \%$ statistical power to detect effect sizes $>2.5$ at a genome-wide significant level and than 1.46 for a $P$-value threshold of 0.05 .

Genotyping frequencies have been deposited at the European Genomephenome Archive (EGA, http://www.ebi.ac.uk/ega/) which is hosted at the EBI, under accession number EGAS00001000734. The genotyping data are also available via the Dutch Biobanking and Biomolecular Research Infrastructure (https://catalogue.bbmri.nl/biobanks/, accession number 199).

\section{Quality-control filtering}

After genome-wide genotyping, a call-rate threshold above $98 \%$ was used for inclusion of the samples. Subjects were excluded from the sample if their sex was inconsistent with genetic data from the $\mathrm{X}$ chromosome and if duplicate samples produced inconsistent genotypes. SNPs were excluded if they had 
(i) successful genotyping in $<90 \%$ of the cases and controls, (ii) a minor allele frequency $<1 \%$ in the population, (iii) showed deviation from HardyWeinberg equilibrium (P-value $<0.0001$ ), or (iv) were monomorphic across all samples. After these exclusions, 535179 SNPs remained.

\section{Genotyping in MARCH and FHFU}

In MARCH and FHFU, the genotypes of selected SNPs were determined using fluorescence-based TaqMan allelic discrimination assays and analyzed on an ABI Prism 7900 Sequence Detection System (Applied Biosystems, Foster City, CA, USA). Reaction components and amplification parameters were based on the manufacturer's instructions using an annealing temperature of $60^{\circ} \mathrm{C}$. Results were scored blinded to CHD status. SNPs were excluded following the same quality criteria as in the gene-finding stage.

\section{Statistical analysis}

The genomic inflation factor was calculated using the mean of the $\chi^{2}$-tests generated on all SNPs that were tested. For each SNP which passed the quality control in the ARGOS population, the association with risk of CHD was examined in an additive genetic model using a logistic regression model adjusted for sex. Given the fact that age was an inclusion criterion to generate the contrast between cases and controls of the ARGOS population, we did not adjust for age.

We selected all SNPs that were associated with $\mathrm{CHD}$ with a $P$-value $<1.00 \times 10^{-4}$ in ARGOS to analyze in the second stage. In the second stage, the association with risk of CHD was examined using logistic regression in MARCH and the FHFU. In MARCH, we adjusted for sex only, as age was a selection criterion similar to ARGOS. In the FHFU cohort, we adjusted for age, sex, and statin use, as statin use was expected to be a confounder and, in contrast to ARGOS and MARCH, it was well documented in that cohort. Using Bonferroni correction, significance threshold was 0.0017 (0.05/29). We performed a z-based meta-analysis to combine the results of MARCH and FHFU in the second stage. Furthermore, we combined the results of all three studies using z-based meta-analysis.

We used Plink version 1.03 to run GWA study in ARGOS, SPSS version 15 to run logistic regression models in MARCH and FHFU and finally 'meta' and 'rmeta' packages running under $\mathrm{R}$ to perform the meta-analysis. ${ }^{15-17}$

\section{Replication of well-known SNPs associated with CHD}

To test the effect of earlier defined genetic risk variants on CHD in ARGOS, we analyzed the SNPs described in a large-scale meta-analysis in the CARDIoGRAM consortium (22 233 cases and 64762 controls). Whenever the SNP was not available on the IlluminaHap550, we used a proxy as identified by SNAP (http://www.broadinstitute.org/mpg/snap/ldsearchs.php).

\section{RESULTS}

\section{Characteristics of the ARGOS cohort}

Out of 17000 Dutch FH patients with a known LDLR mutation, we selected the 264 youngest FH patients with CHD and the 236 oldest $\mathrm{FH}$ patients without any $\mathrm{CHD}$. The mean $\pm \mathrm{SD}$ (range) age was $41.7 \pm 8.3$ (23-59 years) in cases and $75.6 \pm 5.9$ (65-years) in controls. A total of 249 cases and 217 controls were successfully genotyped. There were no significant differences in age, smoking, or plasma cholesterol levels between the patients who were and those who were not successfully genotyped (data not shown). General characteristics of the genotyped patients are shown in Table 1 and the age distribution in Supplementary Figure 1.

Eighty-one cases $(32.5 \%)$ had a negative LDLR mutation, for example, a mutation leading to complete loss of function of the LDL receptor, whereas only 42 controls $(19.4 \%)$ had a receptornegative mutation $(P=0.004)$. This was mainly due to an overrepresentation of the c.1359-1G $>$ A mutation, which was present in 46 cases and only in 21 controls. The other mutations were equally distributed (Supplementary Table 1). On average, the controls were 34 years older than the cases $(P$-value $<0.001)$. Consequently, hypertension and diabetes mellitus were more often present in the controls than in the cases. More cases than controls were ever smokers (Table 1).

\section{Stage I (GWA analysis)}

After quality-control filtering, we included 535179 SNPs in the GWA analysis. A quantile-quantile plot of the observed against expected $P$-value distribution is shown in Figure 2. The genomic inflation factor $(\lambda \mathrm{gc})$ was 1.01 in the total sample. Supplementary Figure 2 illustrates the primary findings from the GWA analysis in the ARGOS population and presents $P$-values for each of the interrogated SNPs across the chromosomes. For a total of 40 SNPs clustered around 21 loci on all chromosomes except 3, 6, 12, 15, 16, and 18-21 the $P$-value was lower than the threshold of $1 \times 10^{-4}$ (Table 2). Of these, 11 were in complete linkage disequilibrium (LD) with a leading SNP in the same locus. Twelve out of 40 SNPs were located in a cluster on chromosome 11p15; they were located in four different LD blocks and could be tagged by six SNPs. We took 29 SNPs, including the 6 SNPs in $11 \mathrm{p} 15$, to stage II.

Table 1 Characteristics of the ARGOS population and the replication populations

Diabetes

\begin{tabular}{|c|c|c|c|c|c|c|c|}
\hline Population & $N$ & Males (\%) & Age (years) & Smoking (\%) & Mellitus (\%) & Hypertension (\%) & Total Cholesterol $(\mathrm{mmol} / \mathrm{l})$ \\
\hline \multicolumn{8}{|c|}{ Stage I: genome-wide analysis } \\
\hline \multicolumn{8}{|c|}{ ARGOS } \\
\hline $\mathrm{CHD}$ cases & 249 & 55.0 & $41.7 \pm 8.4^{\mathrm{a}}$ & $74.2^{\mathrm{a}}$ & $2.4^{\mathrm{a}}$ & $19.7^{a}$ & $11.3 \pm 2.6^{a}$ \\
\hline Controls & 217 & 47.9 & $75.6 \pm 5.9^{a}$ & $51.2^{\mathrm{a}}$ & $9.2^{\mathrm{a}}$ & $30.0^{\mathrm{a}}$ & $10.6 \pm 2.8^{a}$ \\
\hline \multicolumn{8}{|c|}{ Stage II: replication } \\
\hline \multicolumn{8}{|c|}{$\mathrm{MARCH}$} \\
\hline CHD cases & 190 & 63.2 & $39.4 \pm 7.4$ & $49.5^{b}$ & 10.0 & 36.3 & $11.0 \pm 2.2^{\mathrm{a}}$ \\
\hline Controls & 223 & 60.1 & $63.6 \pm 9.2$ & $47.1^{\mathrm{b}}$ & 11.2 & 34.5 & $10.5 \pm 1.7^{a}$ \\
\hline \multicolumn{8}{|l|}{ FHFU } \\
\hline CHD cases & 554 & $65.5^{a}$ & $48.9 \pm 10.6^{a}$ & $83.0^{\mathrm{a}}$ & $4.8^{\mathrm{a}}$ & $16.7^{a}$ & $9.5 \pm 2.1$ \\
\hline Controls & 1468 & $43.5^{a}$ & $46.6 \pm 12.7^{a}$ & $70.3^{a}$ & $1.7^{a}$ & $6.0^{\mathrm{a}}$ & $9.4 \pm 1.9$ \\
\hline
\end{tabular}

Abbreviations: $\mathrm{CHD}$, coronary heart disease; $\mathrm{N}$, number of participants.

Continuous variables are given as mean $\pm \mathrm{SD}$.

a $P<0.05$ for the difference between CHD cases and controls within the cohort.

${ }^{b}$ Cases and controls were matched for smoking in the MARCH Study. 
Stage II

We successfully genotyped 28 SNPs in MARCH and all 29 SNPs in FHFU (Supplementary Table 2). For none of the SNPs, the $P$-value was lower than the Bonferroni corrected threshold of 0.017 either in MARCH or FHFU. The smallest $P$-value was found for rs176388 (odds ratio OR 0.33, $P=0.042$ ) in $\mathrm{MARCH}$. Although the direction of the effect was the same in FHFU, the association was not significant (OR $0.80, P=0.36$ ). We performed a meta-analysis to combine the results of the analysis in MARCH and FHFU. None of the SNPs

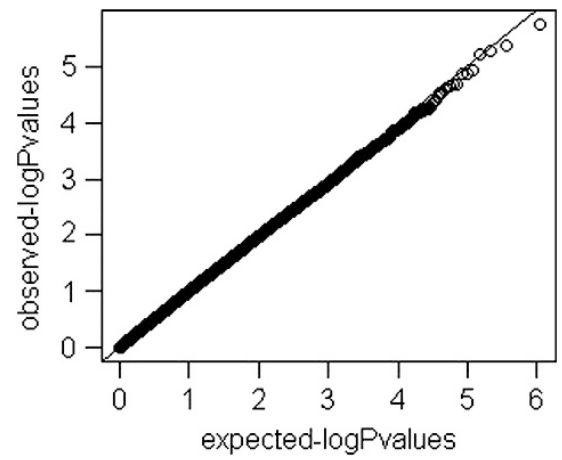

Figure 2 QQ Plot. reached the Bonferroni significant threshold after combining the results of MARCH and FHFU.

Finally, we combined the results of ARGOS, MARCH, and FHFU; however, none of the top SNPs identified in ARGOS reached the genome-wide significance level. The smallest combined $P$-value was $4 \times 10^{-4}$ for rs176388, for which the A allele showed a protective effect in all cohorts. Second best was rs1380945, for which all cohorts showed that the $\mathrm{G}$ allele was associated with increased $\mathrm{CHD}$ risk (Table 3 and Supplementary Figure 3).

Genotyping additional cohorts from the general population did not show any genome-wide significance.

\section{Replication of well-known SNPs associated with CHD in the general population}

To examine if the genetic risk variants identified in GWA studies in the general population also showed an effect in ARGOS and to test whether effect sizes were larger in our 'extreme genetics' population, we examined the association of 25 previously reported genetic risk factors in CARDIoGRAM. In this meta-analysis, 9 out of 12 previously reported $\mathrm{CHD}$ loci were confirmed with a $P$-value of $<5.0 \times 10^{8}$ and 13 new ones identified (Table 4). ${ }^{5}$ None of the studied SNPs were significantly associated with CHD in ARGOS. Lowest $P$-values were obtained for rs4977574 at 9p21 (CARDIoGRAM OR $1.29,95 \%$ CI $1.23-1.36 ; \quad P=1.35 \times 10^{-22}$;

Table 2 SNPs influencing coronary heart disease risk in the ARGOS population (with $P<1.0 \times 10^{-4}$ )

\begin{tabular}{|c|c|c|c|c|c|c|c|c|c|c|c|c|}
\hline$S N P$ & Chr & Position & $\begin{array}{c}\text { Accession } \\
\text { number }\end{array}$ & $\begin{array}{c}\text { Reference } \\
\text { allele }\end{array}$ & $\begin{array}{c}\text { Alternate } \\
\text { allele }\end{array}$ & SNP type & Gene & $\begin{array}{l}\text { Freq } \\
\text { cases }\end{array}$ & $\begin{array}{c}\text { Freq } \\
\text { controls }\end{array}$ & $O R$ & $95 \% \mathrm{Cl}$ & $\mathrm{P}$-value \\
\hline rs12087224 & $1 \mathrm{p} 22$ & 83902972 & NT_032977.8 & G & A & Intergenic & TTLL7 & 0.15 & 0.06 & 2.54 & $1.59-4.04$ & $8.86 \times 10^{-5}$ \\
\hline rs7581691 & $2 p 24$ & 13953005 & NT_005334.15 & G & $A$ & - & - & 0.16 & 0.07 & 2.62 & $1.67-4.11$ & $2.93 \times 10^{-5}$ \\
\hline rs1406333 & $2 q 32$ & 184155187 & NT_005403.16 & $A$ & G & Intergenic & NUP35 & 0.33 & 0.47 & 0.59 & $0.45-0.77$ & $8.23 \times 10^{-5}$ \\
\hline rs2412397 & $4 q 12$ & 53804380 & NT_022853.14 & $A$ & G & Intronic & SCFD2 & 0.31 & 0.44 & 0.57 & $0.43-0.76$ & $9.98 \times 10^{-5}$ \\
\hline rs778940 & $4 q 13$ & 62926680 & NT_022778.15 & $A$ & G & Intergenic & LPHN3 & 0.16 & 0.07 & 2.44 & $1.57-3.77$ & $6.74 \times 10^{-5}$ \\
\hline rs6835823 & $4 q 32$ & 155191807 & NT_016354.18 & $A$ & C & Intergenic & DCHS2 & 0.48 & 0.37 & 1.77 & $1.36-2.30$ & $2.11 \times 10^{-5}$ \\
\hline rs4696207 & $4 q 32$ & 155488838 & NT_016354.18 & C & $A$ & Intronic & DCHS2 & 0.50 & 0.36 & 1.80 & $1.36-2.38$ & $3.36 \times 10^{-5}$ \\
\hline rs7691894 & $4 q 32$ & 167900239 & NT_022792.17 & $A$ & G & Intronic & SPOCK3 & 0.39 & 0.23 & 2.06 & $1.53-2.77$ & $1.74 \times 10^{-6}$ \\
\hline rs11950716 & $5 p 15.2$ & 14141834 & NT_006576.15 & G & $A$ & Intergenic & TRIO & 0.14 & 0.25 & 0.51 & $0.36-0.71$ & $9.45 \times 10^{-5}$ \\
\hline rs17638888 & 7q22 & 98335561 & NT_007933.14 & G & $A$ & Intronic & TRRAP & 0.03 & 0.09 & 0.27 & $0.14-0.51$ & $5.89 \times 10^{-5}$ \\
\hline rs12544799 & $8 q 24.2$ & 130732692 & NT_008046.15 & $A$ & G & Intergenic & $M L Z E$ & 0.41 & 0.28 & 1.87 & $1.40-2.50$ & $2.53 \times 10^{-5}$ \\
\hline rs6985166 & $8 q 24.2$ & 130748358 & NT_008046.15 & $A$ & G & Intergenic & $M L Z E$ & 0.38 & 0.26 & 1.81 & $1.35-2.43$ & $7.81 \times 10^{-5}$ \\
\hline rs13291498 & 9p21 & 28326185 & NT_008413.17 & $A$ & C & Intronic & LINGO2 & 0.21 & 0.33 & 0.55 & $0.41-0.73$ & $5.44 \times 10^{-5}$ \\
\hline rs10761805 & 10q21 & 65294147 & NT_008583.16 & G & $A$ & Intergenic & REEP3 & 0.49 & 0.35 & 1.89 & $1.42-2.51$ & $1.36 \times 10^{-5}$ \\
\hline rs955353 & $10 q 21$ & 65314593 & NT_008583.16 & $\mathrm{G}$ & $A$ & - & - & 0.37 & 0.50 & 0.57 & $0.43-0.75$ & $7.20 \times 10^{-5}$ \\
\hline rs2111995 & $10 q 25$ & 107497352 & NT_030059.12 & $A$ & $\mathrm{G}$ & Intergenic & SORCS3 & 0.28 & 0.17 & 1.97 & $1.42-2.72$ & $4.10 \times 10^{-5}$ \\
\hline rs2647547 & $11 \mathrm{p} 15$ & 5359744 & NT_009237.17 & A & C & Intergenic & OR51M1 & 0.46 & 0.33 & 1.75 & $1.33-2.31$ & $5.85 \times 10^{-5}$ \\
\hline rs1532514 & $11 \mathrm{p} 15$ & 5373198 & NT_009237.17 & C & $A$ & Intergenic & OR51M1 & 0.48 & 0.38 & 1.71 & $1.32-2.23$ & $6.37 \times 10^{-5}$ \\
\hline rs10838092 & $11 \mathrm{p} 15$ & 5400443 & NT_009237.17 & $\mathrm{G}$ & $A$ & Exonic & OR51Q1 & 0.45 & 0.31 & 1.73 & $1.32-2.26$ & $6.73 \times 10^{-5}$ \\
\hline rs10838102 & $11 \mathrm{p} 15$ & 5414207 & NT_009237.17 & G & $A$ & Intergenic & OR51/1 & 0.46 & 0.32 & 1.78 & $1.36-2.33$ & $3.06 \times 10^{-5}$ \\
\hline rs1498486 & $11 p 15$ & 5418567 & NT_009237.17 & $A$ & C & Exonic & OR51I1 & 0.48 & 0.34 & 1.81 & $1.38-2.38$ & $2.02 \times 10^{-5}$ \\
\hline rs2133235 & $11 \mathrm{p} 15$ & 5423352 & NT_009237.17 & $\mathrm{C}$ & A & Intergenic & OR51I1 & 0.44 & 0.30 & 1.82 & $1.28-2.40$ & $2.27 \times 10^{-5}$ \\
\hline rs2846186 & $11 q 25$ & 134203586 & NT_033899.7 & $A$ & G & Intergenic & B3GAT1 & 0.18 & 0.30 & 0.50 & $0.36-0.68$ & $1.18 \times 10^{-5}$ \\
\hline rs2661969 & $11 q 25$ & 134223427 & NT_033899.7 & A & G & Intergenic & B3GAT1 & 0.18 & 0.29 & 0.54 & $0.39-0.73$ & $9.16 \times 10^{-5}$ \\
\hline rs1380945 & $13 q 13$ & 31739619 & NT_024524.13 & $A$ & G & Intronic & FRY & 0.48 & 0.35 & 1.74 & $1.32-2.30$ & $9.76 \times 10^{-5}$ \\
\hline rs4982548 & $14 q 11$ & 21605911 & NT_026437.11 & G & $A$ & Intergenic & OR4E2 & 0.43 & 0.57 & 0.56 & $0.42-0.73$ & $2.43 \times 10^{-5}$ \\
\hline rs2531851 & $17 p 13$ & 9141140 & NT_010718.15 & G & $A$ & Intronic & STX8 & 0.40 & 0.27 & 1.92 & $1.43-2.59$ & $1.40 \times 10^{-5}$ \\
\hline rs5755595 & $22 q 12$ & 33848839 & NT_011520.11 & G & $A$ & Intergenic & $R A X L X$ & 0.38 & 0.26 & 1.84 & $1.36-2.48$ & $6.28 \times 10^{-5}$ \\
\hline rs5928090 & Xp21 & 32780853 & NT_011757.15 & $A$ & G & - & - & 0.42 & 0.27 & 1.75 & $1.18-2.60$ & $4.20 \times 10^{-5}$ \\
\hline
\end{tabular}

Abbreviations: $\mathrm{Cl}$, confidence interval; $\mathrm{Chr}$, chromosome; Freq, frequency; OR, odds ratio; SNP, single nucleotide polymorphism.

Chromosome base position according to NCBI Genome build 36.3. Intergenic is defined as $<500 \mathrm{Mb}$ from the gene. 
Table 3 Results of the genome-wide association study in the two $\mathrm{FH}$ replication populations

\begin{tabular}{|c|c|c|c|c|c|c|c|c|c|}
\hline \multirow[b]{2}{*}{ SNP } & \multirow[b]{2}{*}{ Risk allele } & \multicolumn{2}{|c|}{$M A R C H(n=413)$} & \multicolumn{2}{|c|}{ FHFU $(\mathrm{N}=2022)$} & \multicolumn{2}{|c|}{ Meta-analysis MARCH + FHFU } & \multicolumn{2}{|c|}{ Meta-analysis ARGOS + MARCH + FHFU } \\
\hline & & $O R$ & P-value & $O R$ & P-value & $O R$ & P-value & $O R$ & P-value \\
\hline rs7581691 & A & 1.07 & 0.79 & 0.86 & 0.35 & 0.91 & 0.51 & 1.20 & $1.2 \times 10^{-1}$ \\
\hline rs1406333 & G & 0.98 & 0.89 & 0.96 & 0.71 & 0.98 & 0.68 & 0.84 & $1.5 \times 10^{-2}$ \\
\hline rs2412397 & G & NA & NA & 0.91 & 0.36 & NA & NA & NA & NA \\
\hline rs4696207 & $A$ & 0.90 & 0.48 & 1.10 & 0.33 & 1.03 & 0.70 & 1.19 & $1.4 \times 10^{-2}$ \\
\hline rs7691894 & $G$ & 1.02 & 0.99 & 1.03 & 0.74 & 1.02 & 0.80 & 1.20 & $1.2 \times 10^{-2}$ \\
\hline rs11950716 & $A$ & 0.83 & 0.35 & 0.94 & 0.60 & 0.91 & 0.34 & 0.78 & $5.8 \times 10^{-3}$ \\
\hline rs17638888 & A & 0.52 & 0.04 & 0.80 & 0.36 & 0.69 & 0.05 & 0.54 & $2.0 \times 10^{-4}$ \\
\hline rs12544799 & $G$ & 1.06 & 0.70 & 0.94 & 0.52 & 0.98 & 0.77 & 1.14 & $7.6 \times 10^{-2}$ \\
\hline rs6985166 & G & 1.05 & 0.76 & 1.09 & 0.38 & 1.08 & 0.36 & 1.22 & $6.4 \times 10^{-3}$ \\
\hline rs2647547 & C & 0.98 & 0.90 & 1.04 & 0.67 & 1.02 & 0.79 & 1.17 & $2.4 \times 10^{-2}$ \\
\hline rs1532514 & $A$ & 1.04 & 0.79 & 1.01 & 0.88 & 1.02 & 0.81 & 1.16 & $2.6 \times 10^{-2}$ \\
\hline rs10838092 & A & 1.09 & 0.59 & 1.00 & 0.96 & 1.02 & 0.80 & 1.18 & $2.4 \times 10^{-2}$ \\
\hline rs10838102 & A & 1.20 & 0.21 & 0.96 & 0.70 & 1.03 & 0.71 & 1.19 & $1.5 \times 10^{-2}$ \\
\hline rs1498486 & C & 1.11 & 0.45 & 1.07 & 0.51 & 1.08 & 0.34 & 1.23 & $3.1 \times 10^{-3}$ \\
\hline rs2133235 & A & 1.20 & 0.22 & 1.93 & 0.48 & 1.01 & 0.93 & 1.17 & $2.5 \times 10^{-2}$ \\
\hline rs2846186 & G & 0.83 & 0.26 & 1.06 & 0.63 & 0.97 & 0.76 & 0.81 & $1.1 \times 10^{-2}$ \\
\hline rs2661969 & G & 0.83 & 0.23 & 0.97 & 0.97 & 0.91 & 0.35 & 0.79 & $4.9 \times 10^{-3}$ \\
\hline rs1380945 & G & 1.25 & 0.13 & 1.14 & 0.19 & 1.17 & 0.06 & 1.29 & $3.0 \times 10^{-4}$ \\
\hline rs4982548 & A & 1.02 & 0.89 & 1.07 & 0.45 & 1.05 & 0.48 & 0.91 & $1.7 \times 10^{-1}$ \\
\hline rs2531851 & A & 1.07 & 0.63 & 0.89 & 0.23 & 0.95 & 0.51 & 1.11 & $1.4 \times 10^{-1}$ \\
\hline rs5755595 & A & 0.80 & 0.15 & 0.90 & 0.33 & 0.86 & 0.10 & 1.05 & $5.4 \times 10^{-1}$ \\
\hline
\end{tabular}

Abbreviation: OR, odds ratio.

Combined $P$-value given for replication populations.

ARGOS OR 1.28, 95\% CI 1.00-1.67; $P=0.05)$ and for the SNP in the proprotein convertase subtilisin/kexin type 9 (PCSK9) gene that did not show genomic signficance in CARDIoGRAM (CARDIoGRAM OR $1.08,95 \%$ CI $1.05-1.11 ; P=9.1 \times 10^{-8}$; ARGOS OR $1.52,95 \%$ CI $1.10-2.12, P=0.01)$.

\section{DISCUSSION}

In this study, by using an extreme genetics approach, we aimed to fortify our statistical power to identify novel genetic risk variants for CHD. However, none of the suggestive findings were either confirmed in the second stage or reached the genome-wide significant threshold in a meta-analysis of all populations combined.

We expected to identify larger effect sizes in our GWA study, compared with traditional GWA studies on CHD, for two reasons. First, we studied genetic risk variants for $\mathrm{CHD}$ in a cohort of $\mathrm{FH}$ patients, and hypercholesterolemia is one of the most important risk factors for CHD. Based on Rothman's model of causation, one would expect that in the presence of similar environmental factors, risk variants in genes will be associated with larger outcome effects. ${ }^{6}$ Second, we applied an extreme selection approach. Therefore, the genetic contrast between cases and controls was expected to increase. ${ }^{18}$ This incremental contrast has been shown to increase the power in simulation studies in quantitative traits. The effect sizes found when selectively genotyping only the phenotypic extremes will be increased. ${ }^{18-20}$ Plomin suggested that common disorders could also be considered as quantitative traits, as risk on a common disease in the population could be regarded as a distribution of 'polygenetic liability' to a disease. The extreme sampling approach should, therefore, produce larger effect sizes in our study as well. Thus, the power was expected to be relatively high, despite the reduction in number of individuals as a result of the selection criteria. ${ }^{18,19,21}$ Our study is the first to apply this method using real data. Using Quanto, we confirmed that the study was sufficiently powered to identify risk variants with large effect sizes in this high-risk group of subjects. Our findings, being more specific our odds ratios, indicate that the leverage in effect size using this approach is, in fact, quite modest. This is also clear from the data from CARDIoGRAM in our study, as an example, the well-replicated locus at 9p21.3 had an OR of 1.28, very close to the 1.25 value that was found in the original study of subjects from the general population. Most other known CHD risk SNPs identified in earlier studies and published by Schunkert et al ${ }^{5}$ were not associated with statistically significant effects in this $\mathrm{FH}$ sample. However, our study was underpowered for this analysis if the true effect sizes were the same as reported and less increased by our design than anticipated, so we can only look at the direction of the effect. Out of 18 SNPs that could be tested, 11 ORs were in the same direction, whereas only 6 were not (Table 4 ). Of these 11 , most interesting was the SNP in PCSK9. This SNP is associated with an $8 \%$ higher risk of CHD in the general population; however, the odds in ARGOS were increased by $>50 \%$. Although the $P$-value in 
Table 4 Results of earlier identified genetic risk factors in ARGOS 5

CARDIOGRAM

\begin{tabular}{|c|c|c|c|c|c|c|c|c|c|c|c|}
\hline SNP & Chr & $\begin{array}{l}\text { Gene(s) in } \\
\text { region }\end{array}$ & $\begin{array}{c}\text { Accession } \\
\text { number }\end{array}$ & $\begin{array}{l}\text { Ref. } \\
\text { allele }\end{array}$ & $\begin{array}{l}\text { Risk allele } \\
\text { (frequency) }\end{array}$ & $O R$ & P-value & Genotyped & Proxy $^{a}$ & $O R^{\mathrm{b}}$ & P-value \\
\hline rs11206510 & $1 \mathrm{p} 32.3$ & PCSK9 & NT_032977.9 & c & $\mathrm{T}(0.82)$ & 1.08 & $9.1 \times 10^{-8}$ & Yes & & 1.52 & 0.01 \\
\hline rs599839 & $1 \mathrm{p} 13.3$ & SORT1 & NT_019273.18 & G & $A(0.78)$ & 1.11 & $2.9 \times 10^{-10}$ & No & rs646776 & 1.00 & 0.99 \\
\hline rs17465637 & $1 \mathrm{q} 41$ & MIA3 & NT_021877.18 & A & $C(0.74)$ & 1.14 & $1.4 \times 10^{-8}$ & No & No proxy & & \\
\hline rs6725887 & $2 \mathrm{q} 33.1$ & WDR12 & NT_005403.16 & $\mathrm{T}$ & $C(0.15)$ & 1.14 & $1.1 \times 10^{-9}$ & Yes & & 1.07 & 0.76 \\
\hline rs17609940c & $6 \mathrm{p} 21.31$ & ANKS1A & NT_007592.14 & C & $G(0.75)$ & 1.07 & $2.2 \times 10^{-6}$ & No & rs820082 & 0.83 & 0.25 \\
\hline rs12190287c & $6 q 23.2$ & TCF21 & NT_025741.14 & G & $C(0.62)$ & 1.08 & $4.6 \times 10^{-11}$ & No & No proxy & & \\
\hline rs3798220 & $6 q 25.3$ & $\angle P A$ & NT_007422.13 & $\mathrm{T}$ & $C(0.02)$ & 1.09 & $3.0 \times 10^{-11}$ & No & No LD data & & \\
\hline rs11556924c & $7 q 32.2$ & $\mathrm{ZC} 3 \mathrm{HCl}$ & NT_007933.14 & $\mathrm{T}$ & $C(0.62)$ & 1.09 & $2.2 \times 10^{-9}$ & No & No proxy & & \\
\hline rs4977574 & $9 p 21.3$ & CDKN2A, CDKN2B & NT_008413.17 & A & $G(0.46)$ & 1.25 & $1.4 \times 10^{-22}$ & Yes & & 1.28 & 0.05 \\
\hline rs579459c & $9 q 34.2$ & $A B O$ & NT_035014.4 & $\mathrm{T}$ & $C(0.21)$ & 1.10 & $1.2 \times 10^{-7}$ & No & rs495828 & 1.35 & 0.08 \\
\hline rs4773144c & $13 q 34$ & COLAA1, COL4A2 & NT_009952.14 & A & $G(0.44)$ & 1.07 & $4.2 \times 10^{-7}$ & No & No proxy & & \\
\hline rs2895811 c & $14 \mathrm{q} 32.2$ & HHIPLI & NT_026437.11 & $\mathrm{T}$ & $C(0.43)$ & 1.07 & $2.7 \times 10^{-7}$ & Yes & & 1.05 & 0.72 \\
\hline rs3825807c & $15 q 25.1$ & ADAMTS7 & NT_01094.16 & G & $A(0.57)$ & 1.08 & $9.6 \times 10^{-6}$ & No & rs7177699 & NA & NA \\
\hline rs216172c & $17 p 13.3$ & SMG6, SRR & NT_010718.15 & G & $C(0.37)$ & 1.07 & $6.2 \times 10^{-7}$ & No & rs2281727 & 0.85 & 0.26 \\
\hline rs12936587c & $17 p 11.2$ & RASD1, SMCR3, PEMT & NT_010718.15 & A & $G(0.56)$ & 1.07 & $4.9 \times 10^{-7}$ & No & rs11871738 & 0.83 & 0.14 \\
\hline rs $46522^{c}$ & $17 q 21.32$ & UBE2Z, GIP, ATP5G1, SNF8 & NT_010783.14 & C & $\mathrm{T}(0.53)$ & 1.06 & $3.6 \times 10^{-6}$ & No & rs962272 & 1.04 & 0.73 \\
\hline rs1122608 & $19 p 13.2$ & $L D L R$ & NT_011295.10 & $\mathrm{T}$ & $G(0.77)$ & 1.14 & $9.7 \times 10^{-10}$ & No & rs3786725 & 0.81 & 0.17 \\
\hline rs9982601 & $21 q 22.11$ & MRPS6 & NT_011512.10 & C & $\mathrm{T}(0.15)$ & 1.18 & $4.2 \times 10^{-10}$ & No & rs7278204 & 1.28 & 0.19 \\
\hline
\end{tabular}

Abbreviation: Ref., reference.

Chromosome position according to NCBI Genome build 36.3 .

aUsing SNAP (http://www. broadinstitute.org/mpg/snap/ldsearchs. php).

bisk allele or representative proxy.

'OR representing combined analysis CARDIoGRAM \& replication cohort. ${ }^{5}$

CARDIoGRAM did not reach genome-wide significance, it is tempting to speculate that this finding and the enhanced effect in ARGOS is not due to chance, but might reflect an interaction between this gene and cholesterol levels. PCSK9 is a gene encoding proprotein convertase subtilisin/kexin type 9, involved in the intracellular degradation of LDL receptors. PCSK9 levels correlated inversely with LDL cholesterol levels in $\mathrm{FH}$ patients. ${ }^{22}$ PCSK9 inhibitors are being tested in phase III trials ${ }^{23}$ now, and are expected to be highly effective in $\mathrm{FH}$ patients. ${ }^{24,25}$

Our definition of extreme groups might have been too restrictive, as it constitutes a very small proportion of the $\mathrm{FH}$ sample (500 out of 17000 ).

Nevertheless, our findings may help to understand why genetic risk prediction models have not yet succeeded. In our approach, we attempted to identify novel genes by selecting the extreme groups of the CHD risk distribution spectrum. Genetic risk prediction studies, on the other hand, start with genetic information and estimate who will end up in the low- and the high-risk group. ${ }^{26}$ As we could demonstrate that the effect sizes in this design are not as enlarged as we expected, the contrast needed for prediction might be less than anticipated as well: genetic risk factors most likely have a more gradual distribution over the population instead of being over- or underrepresented in the phenotypic extremes and the combination of classical and genetic risk factors defines the CHD risk.

Our approach has a number of limitations. Finding extreme cases is a challenging effort. Although $\mathrm{FH}$ is a relatively common genetic disorder, collecting a large sample of subjects either with early onset CHD or healthy aging is difficult. The low genomic inflation factor (1.01) in the total sample indicated that population admixture was not likely. ${ }^{27}$ We do realize that from a statistical point of view our sample size was not enough to detect genes with small effects. Another issue might be whether difference in age between cases and controls might bring in extra confounding. Although only Caucasian patients were studied, first and second degree family members were excluded and we had phenotypic data, we cannot exclude the presence of an unknown confounder. A last limitation of a GWA study in FH subjects is that the results may not necessarily apply to the general population. $^{28}$ Results may be restricted to $\mathrm{FH}$ patients or to hypercholesterolemic patients in general. Also, it might be possible that $\mathrm{CHD}$ risk in the young is genetically different from CHD risk at an older age.

We conclude that the genetics of CHD risk in FH is complex and even applying an 'extreme genetics' approach, we did not identify new genetic risk variants. Most likely, this method is not as effective in leveraging effect size as anticipated, and may, therefore, not lead to significant gains in statistical power. Also, this study might explain why genetic risk prediction modeling is yielding disappointment. The odds ratio associated with genetic variation at the PCSK9 locus points to important consequences for PCSK9 activity in FH patients and provides hope for the novel approach to lower these levels through monoclonal antibodies in order to prevent CHD. 


\section{CONFLICT OF INTEREST}

Professor Kastelein is a recipient of the Lifetime Achievement Award of the Dutch Heart Foundation. Keith J Johnson was an employé of Pfizer in Groton, CT, USA. The funders had no role in study design, data collection and analysis, decision to publish, or preparation of the manuscript. All other authors declare no conflict of interest.

\section{ACKNOWLEDGEMENTS}

We thank Carl Jarman and David Gunn for help in sample preparation and data collection. CIBERobn is an initiative of ISCIII, Spain. This work was supported by the Dutch Heart Foundation (2006B190) and Unilever, UK. Additional funding was provided by Pfizer and MSD The Netherlands. SEH acknowledges BHF support (RG 2008/008) and also funding from the Department of Health's NIHR Biomedical Research Centre funding scheme. ER is supported by grant FIS PS09/01292 from ISCIII, Spain.

1 Rosamond W, Flegal K, Furie K et al: Heart disease and stroke statistics-2008 update: a report from the American Heart Association Statistics Committee and Stroke Statistics Subcommittee. Circulation 2008; 117: e25-146.

2 Peden JF, Farrall M: Thirty-five common variants for coronary artery disease: the fruits of much collaborative labour. Hum Mol Genet 2011; 20: R198-R205.

3 So HC, Gui AH, Cherny SS, Sham PC: Evaluating the heritability explained by known susceptibility variants: a survey of ten complex diseases. Genet Epidemiol 2011; 35: 310-317.

4 Kathiresan S, Voight BF, Purcell S et al: Genome-wide association of early-onset myocardial infarction with single nucleotide polymorphisms and copy number variants. Nat Genet 2009; 41: 334-341.

5 Schunkert H, Konig IR, Kathiresan S et al: Large-scale association analysis identifies 13 new susceptibility loci for coronary artery disease. Nat Genet 2011; 43: 333-338.

6 Rothman KJ, Greenland S: Causation and causal inference in epidemiology. Am J Public Health 2005; 95:Suppl 1 S144-S150.

7 Hobbs HH, Brown MS, Goldstein JL: Molecular genetics of the LDL receptor gene in familial hypercholesterolemia. Hum Mutat 1992; 1: 445-466.

8 McCarthy MI, Abecasis GR, Cardon LR et al: Genome-wide association studies for complex traits: consensus, uncertainty and challenges. Nat Rev Genet 2008; 9: 356-369.

9 Umans-Eckenhausen MA, Defesche JC, Scheerder RL, Cline F, Kastelein JJ: [Tracing of patients with familial hypercholesterolemia in the Netherlands]. Ned Tijdschr Geneeskd 1999; 143: 1157-1161.
10 Jansen AC, van Aalst-Cohen ES, Hutten BA, Buller HR, Kastelein JJ, Prins MH: Guidelines were developed for data collection from medical records for use in retrospective analyses. J Clin Epidemiol 2005; 58: 269-274.

11 Jansen AC, van Aalst-Cohen ES, Tanck MW et al: The contribution of classical risk factors to cardiovascular disease in familial hypercholesterolaemia: data in 2400 patients. J Int Med 2004; 256: 482-490.

12 Hofman A, Breteler MM, van Duijn CM et al: The Rotterdam Study: 2010 objectives and design update. Eur J Epidemiol 2009; 24: 553-572.

13 Helgadottir A, Thorleifsson G, Manolescu A et al: A common variant on chromosome 9p21 affects the risk of myocardial infarction. Science 2007; 316: 1491-1493.

14 Erdmann J, Grosshennig A, Braund PS et al: New susceptibility locus for coronary artery disease on chromosome 3q22.3. Nat Genet 2009; 41: 280-282.

15 Purcell S, Neale B, Todd-Brown K et al: PLINK: a tool set for whole-genome association and population-based linkage analyses. Am J Hum Genet 2007; 81: 559-575.

16 Schwarzer G: The Meta Package. wwwcranr-projectorg/src/contrib/Descriptions/rmetahtm/ 2005.

17 Lumley T: The rmeta package. http://wwwcranr-projectorg/src/contrib/Descriptions/ rmetahtm/ 2004.

18 Van Gestel S, Houwing-Duistermaat JJ, Adolfsson R, van Duijn CM, Van Broeckhoven C: Power of selective genotyping in genetic association analyses of quantitative traits. Behav Genet 2000; 30: 141-146.

19 Xing C, Xing G: Power of selective genotyping in genome-wide association studies of quantitative traits. BMC Proc 2009; 3(Suppl 7): S23.

20 Huang BE, Lin DY: Efficient association mapping of quantitative trait loci with selective genotyping. Am J Hum Genet 2007; 80: 567-576.

21 Plomin R, Haworth CM, Davis OS: Common disorders are quantitative traits. Nat Rev Genet 2009; 10: 872-878.

22 Huijgen R, Fouchier SW, Denoun M et al: Plasma levels of PCSK9 and phenotypic variability in familial hypercholesterolemia. J Lipid Res 2012; 53: 979-983.

23 Blom DJ, Hala T, Bolognese M et al: A 52-week placebo-controlled trial of evolocumab in hyperlipidemia. N Engl J Med 2014; 370: 1809-1819.

24 Cohen JC, Boerwinkle E, Mosley Jr TH, Hobbs HH: Sequence variations in PCSK9, low LDL, and protection against coronary heart disease. N Eng/ J Med 2006; 354: 1264-1272.

25 Sjouke B, Kusters DM, Kastelein JJ, Hovingh GK: Familial hypercholesterolemia: present and future management. Curr Cardiol Rep 2011; 13: 527-536.

26 loannidis JP: Prediction of cardiovascular disease outcomes and established cardiovascular risk factors by genome-wide association markers. Circ Cardiovasc Genet 2009; 2: 7-15.

27 Devlin B, Roeder K: Genomic control for association studies. Biometrics 1999; 55: 997-1004.

28 Van der Net JB, Oosterveer DM, Versmissen J et al: Replication study of 10 genetic polymorphisms associated with coronary heart disease in a specific high-risk population with familial hypercholesterolemia. Eur Heart J 2008; 29: 2195-2201.

Supplementary Information accompanies this paper on European Journal of Human Genetics website (http://www.nature.com/ejhg) 